\title{
STAT3 - A key integrator of profibrotic signalling
}

Signal transducer and activator of transcription 3 (STAT3) could be a prime target for treating fibrosis in diseases such as systemic sclerosis (SSc), according to new findings published in Nature Communications. Multiple kinases, including those downstream of transforming growth factor- $\beta$ (TGF $\beta$ ) and IL-6, are thought to contribute to the aberrant activation of fibroblasts observed in fibrotic diseases. "We provide the first evidence that STAT3 serves as a key molecular checkpoint in fibroblasts by integrating and converting signals from these kinases into profibrotic responses," reports Debomita Chakraborty, first author of the study.

The molecular mechanisms that govern aberrant fibroblast activation in fibrosis are incompletely understood; although studies have identified TGF $\beta$ as a key molecule in this process, inhibition of individual pathways downstream of TGF $\beta$ do not completely abrogate its profibrotic effects. STAT3, a transcription factor
4

a number of strategies for targeting STAT3 are currently being tested in clinical trials

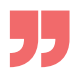

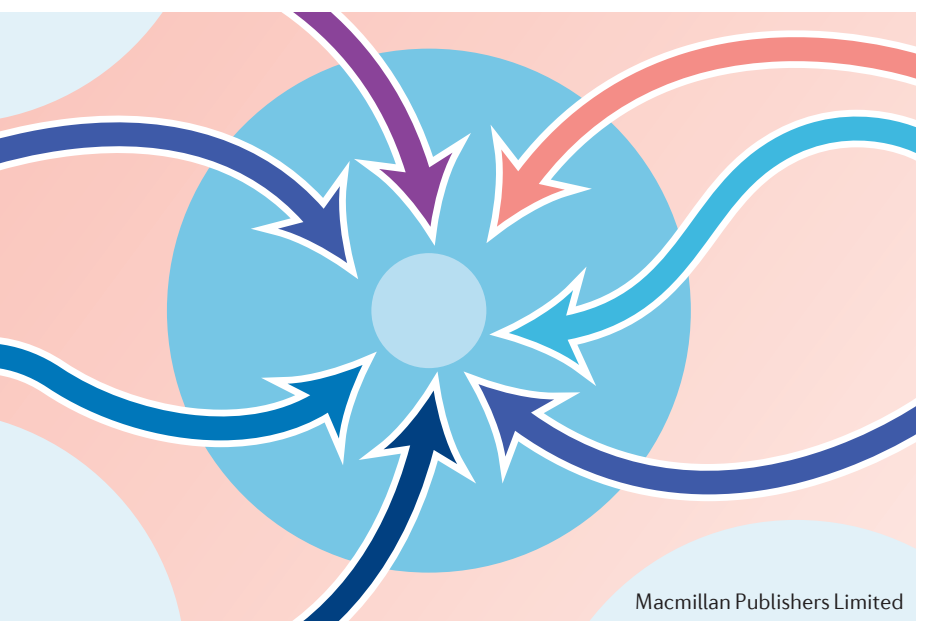

activated downstream of TGF $\beta$, is constitutively expressed in many tissues and cells where it is influenced by various stimuli and has been implicated in the pathogenesis of several diseases. Furthermore, a number of strategies for targeting STAT3 are currently being tested in clinical trials. "These various aspects led us to analyse the activation of STAT3 in SSc and also investigate its role in TGF $\beta$-dependent fibroblast activation and tissue fibrosis," comments corresponding author Jörg Distler.

The researchers observed an increase in the activated (phosphorylated) form of STAT3 (pSTAT3) in tissue from skin biopsies and cultured fibroblasts from patients with SSc compared with age-matched and sex-matched healthy individuals. Recombinant TGF $\beta$ induced the phosphorylation of STAT3 in cultured human fibroblasts, which was associated with the stimulation of several kinases; individual inhibition of these kinases reduced TGF $\beta$-induced phosphorylation of STAT3. Similarly, selective pharmacological inhibition of either TGF $\beta$ or these individual kinases reduced bleomycin-induced phosphorylation of STAT3 in mice.

Blocking STAT3 signalling with a small molecule inhibitor (S31-201) inhibited TGF $\beta$-induced fibroblastto-myofibroblast transition and collagen release in cultured human fibroblasts; similar findings were observed with genetic deletion of Stat 3 in mouse fibroblasts, suggesting that the effects of S31-201 were direct and not due to off-target activity.

"Targeting STAT3, downstream of both ... IL- 6 and TGF $\beta$, would be predicted to be effective in SSc as you are blocking the convergence point," comments Steven O'Reilly, who was not involved with this study. "We have previously demonstrated the importance of IL-6-mediated activation of STAT3 in fibrosis generation," he explains. "Thus blocking STAT3 may block the effects of both cytokines in the disease."

To investigate STAT3 targeting in vivo, Chakraborty et al. used two mouse models of fibrosis: bleomycininduced fibrosis (a model of early, inflammation-driven fibrosis) and fibrosis induced by the overexpression of constitutively active TGF $\beta$ receptor type I (a model of late-stage, non-inflammatory SSc associated with endogenous activation of fibroblasts). Inhibition of STAT3 by either S31-201 or fibroblast-specific genetic inactivation ameliorated fibrosis in both models. "These findings provide proof that STAT3 directly regulates fibroblast activation independently of its well-known effects on inflammation," states Chakraborty.

"The demonstration of direct, inflammation-independent effects on fibroblasts is of high translational relevance given that only one-third of patients with SSc show clinical evidence of inflammation," comments Distler. As SSc is a heterogeneous disease, with variation in the individual upstream profibrotic pathways that are activated, they propose that the identification of such a central signalling nexus could ensure therapeutic effects across different subpopulations of patients.

"We thus demonstrate that STAT3 is a central integrator of multiple profibrotic signals and a promising candidate for molecular targeted therapies of fibrosis in SSc," concludes Chakraborty.

Jessica McHugh

ORIGINAL ARTICLE Chakraborty, D. et al. Activation of STAT 3 integrates common profibrotic pathways to promote fibroblast activation and tissue fibrosis. Nat. Commun. 8, 1130 (2017) 